\title{
Mola Completa em Gravidez Gemelar: Relato de Caso
}

Complete Mole in Twin Pregnancy: a Case Report

Izildinha Maestá ${ }^{1}$, Iracema M.P. Calderon ${ }^{2}$, Marilza V.C. Rudge ${ }^{1}$ Magaly M. Sales ${ }^{3}$, Fabiano P. Saggioro ${ }^{2}$, José Carlos Peraçoli ${ }^{1}$

\begin{abstract}
RESUM0
A gravidez gemelar na qual coexistem um feto normal e uma mola completa é um evento raro. Complicações clínicas e aumento de risco de malignização são de importância nesta patologia. Este trabalho descreve um caso de diagnóstico tardio em decorrência da presença do feto. Este diagnóstico foi feito no momento da resolução da gestação e confirmado por estudo histopatológico e citometria de fluxo. A resolução da gestação foi por via transpélvica em decorrência de hemorragia uterina maciça. O seguimento pós-molar evidenciou a persistência de níveis elevados de bhCG, obtendo-se remissão completa da doença com o uso do metotrexato. À luz deste caso, discutem-se o diagnóstico, a história natural e a conduta desta rara intercorrência na clínica obstétrica.
\end{abstract}

PALAVRAS-CHAVE: Doença trofoblástica gestacional. Gravidez gemelar. Hemorragias da gestação. Quimioterapia. Ultra-sonografia.

\section{Introdução}

A incidência de gravidez gemelar com doença trofoblástica gestacional (DTG) num ovo e gravidez normal no outro ocorre em 1:22.000 a 1:100.000 gestações ${ }^{14}$. O quadro clínico é semelhante ao da DTG, porém, o diagnóstico geralmente é mais tardio pela presença do feto. Os niveis de hCG são mais elevados e é maior a propensão para ocorrência de

\footnotetext{
${ }^{1}$ Disciplina de Obstetrícia, ${ }^{2}$ Disciplina de Patologia, ${ }^{3}$ Divisão Hemocentro, Faculdade de Medicina de Botucatu - UNESP Correspondência:

Izildinha Maestá

Departamento de Ginecologia e Obstetrícia

Faculdade de Medicina de Botucatu - UNESP

18618-000 - Botucatu - SP
}

pré-eclâmpsia e tumor trofoblástico gestacional (TTG). É fundamental o diagnóstico diferencial entre mola hidatiforme parcial com mola completa num ovo e gravidez normal no outro. Na primeira situação o feto apresenta malformações relacionadas à triploidia e cursa com óbito, ao passo que na segunda situação o feto é normal e pode sobreviver. Além disso, o prognóstico materno é bom na mola parcial e é ruim na mola completa com gravidez gemelar. A incidência de TTG após mola completa é de $20 \%$ e após mola parcial é em torno de $5 \%{ }^{4}$. A incidência de TTG em gestação gemelar coexistindo mola completa com feto e placenta normais chega a atingir $55 \%{ }^{14}$.

A chave do diagnóstico da mola completa em gestação gemelar é o cariótipo fetal para excluir 
o seu principal diagnóstico diferencial: mola hidatiforme parcial triplóide. Desde os estudos de citogenética de Kajii e Ohama ${ }^{8}$ e Szulman e Surti ${ }^{15}$ foram definidas as síndromes, mola completa e mola parcial, tal como hoje são entendidas. A origem da doença molar está no mecanismo anormal de fecundação. A mola completa resulta da fertilização de um óvulo que, por motivos totalmente desconhecidos, perde os seus cromossomos, esvazia-se e é fecundado por um espermatozóide que se reproduz por partenogênese, resultando cariótipo diplóide (95\% 46XX; 5\% $46 \mathrm{XY})^{7}$. Portanto, as molas completas são compostas por genoma exclusivamente paterno, ou seja, apresentam natureza androgenética. Na mola parcial o óvulo normal com seus cromossomos é fecundado por 2 espermatozóides determinando um cariótipo triplóide composto por 1 haplóide materno (23X) e 2 paternos ( $46 \mathrm{XX}$ ou $46 \mathrm{XY}$ ). A maior parte das molas parciais apresentam cariótipo $69 \mathrm{XXX}$ ou 69XXY, sendo a dispermia o mecanismo mais freqüente de fertilização.

A gestação gemelar com mola completa num ovo e gravidez normal no outro é considerada dizigótica, em que um gêmeo é normal (46 cromossomos, 23 maternos e 23 de origem paterna) e o outro gêmeo é androgenético ( 46 cromossomos, todos de origem paterna) ${ }^{3}$. A diferença entre diploidia e triploidia, portanto, é importante para determinar as entidades clínicas: mola parcial coexistindo com feto e gestação gemelar com mola completa e feto e placenta normais.

Várias técnicas são utilizadas para a análise cariotípica como a cultura de tecido de vilosidade coriônica obtida por punção. São de importância para recuperação de material de arquivo as técnicas da citometria de fluxo e da hidridização in situ que deteminam o cariótipo a partir de tecido embebido em parafina ${ }^{3,12}$.

Além da diferenciação citogenética, o estudo histopatológico pode diferenciar as sindromes de mola completa (MC) e mola parcial (MP). A presença de âmnio e/ou tecido de origem fetal caracteriza a $\mathrm{MP}$, ao passo que a alteração vilositária difusa sem evidência de feto ocorre na MC.

A despeito das diferenças citogenéticas, as diferenças histopatológicas entre MC e MP podem não ser notadas facilmente, mesmo por patologistas experientes ${ }^{5}$. Em especial, casos de gravidez gemelar com uma mola completa coexistindo com feto podem, realmente, ser, de modo errôneo, classificados como $\mathrm{MP}^{14}$.

A melhor conduta pré-natal nos casos de mola completa em gestação gemelar não está estabelecida na literatura. A análise dos relatos de casos mostra que a interrupção da gravidez é determinada por complicações clínicas decorrentes da agressividade do crescimento do trofoblasto e são caracterizadas por perda de peso materno, hiperêmese gravídica, hemorragia uterina profusa, pré-eclâmpsia e malignização ${ }^{11,16}$. A evolução clínica, laboratorial pelo $\beta$ hCG, a análise citogenética do feto e a decisão da paciente são relevantes ao se considerar a interrupção ou não da gestação.

É apresentado a seguir o relato de caso com as características clínicas e história natural de uma gravidez gemelar coexistindo uma MC e um feto e uma placenta normais. Esse diagnóstico foi confirmado pelo exame histopatológico e pela análise do conteúdo do DNA com a técnica da citometria de fluxo.

\section{Relato do Caso}

Paciente de 18 anos, primigesta nulípara, iniciou seguimento pré-natal sem intercorrências. Por volta da $8^{\mathrm{a}}$ semana, iniciou quadro de hiperêmese gravídica e sangramento uterino intermitente, vermelho vivo, em moderada quantidade, interpretado pelo obstetra e pela ultrasonografia como aborto evitável. Houve persistência da hemorragia genital, náuseas e vômitos, perda de peso, aumento excessivo do tamanho uterino e elevação da pressão arterial materna que atingiu níveis de $170 / 110 \mathrm{mmHg}$. Na $19^{\mathrm{a}}$ semana de gestação foi internada em razão da hemorragia volumosa. Recebeu transfusão de sangue (3 unidades). O exame ultra-sonográfico mostrou presença de feto vivo, cavidade amniótica normal, placenta espessa com inúmeras áreas císticas de tamanho variado recobrindo o orifício interno do colo uterino, suspeitando-se de mola parcial (Figura 1). O estudo da dopplerfluxometria revelou baixa resistência na circulação útero-placentária, com ausência de incisura na artéria uterina (Figura 2). Na propedêutica complementar foram observados aumento sérico do ácido úrico $(5,6 \mathrm{mg} / \mathrm{dl})$, creatinina $(1,2 \mathrm{mg} / \mathrm{dl})$, uréia $(40 \mathrm{mg} / \mathrm{dl}) \mathrm{e}$ proteinúria inferior a $300 \mathrm{mg} / 24$ horas. A dosagem sérica do $\beta$ hCG foi superior a $1.000 .000 \mathrm{mUI} / \mathrm{ml}$. No $2^{\circ}$ dia da admissão, a paciente apresentou contratilidade uterina, cérvico-dilatação de $4 \mathrm{~cm}$, hemorragia uterina grave. Foi realizado esvaziamento uterino imediato, sob anestesia geral, pela técnica de vácuo-aspiração. Após a aspiração da mola foi necessária amniotomia para extração de feto e placenta normais. No momento do parto foi verificado tratar-se de gestação gemelar, na qual coexistem MC num ovo e gravidez normal no outro (Figura 3). O exame histopatológico do tecido molar 
apresentou os critérios clássicos da mola completa, caracterizada por vilos difusamente hidrópicos, com cavitações císticas centrais (Figura 4). A técnica de citometria de fluxo foi realizada para a análise da ploidia do tecido molar. Dificuldades

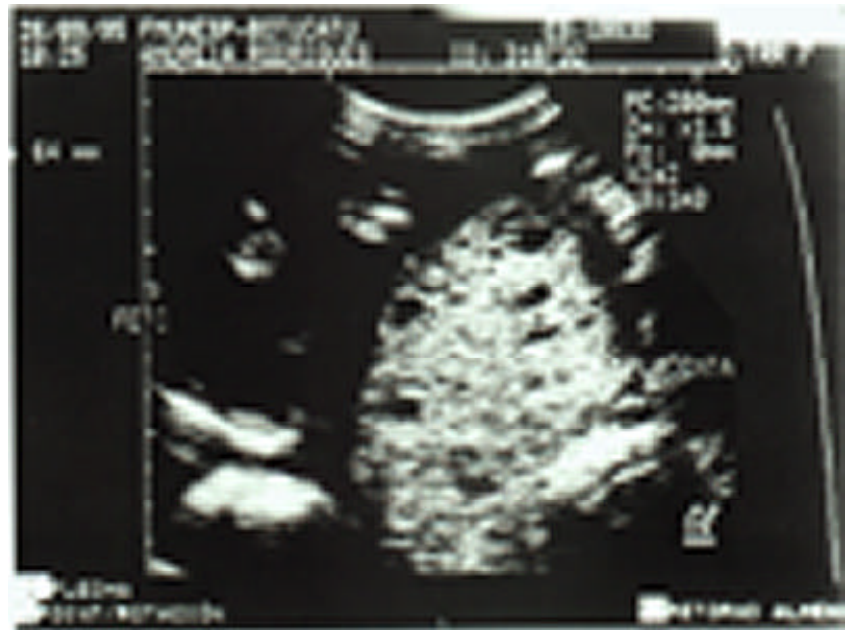

Figura 1- Ultra-som obstétrico mostrando partes fetais e placenta molar

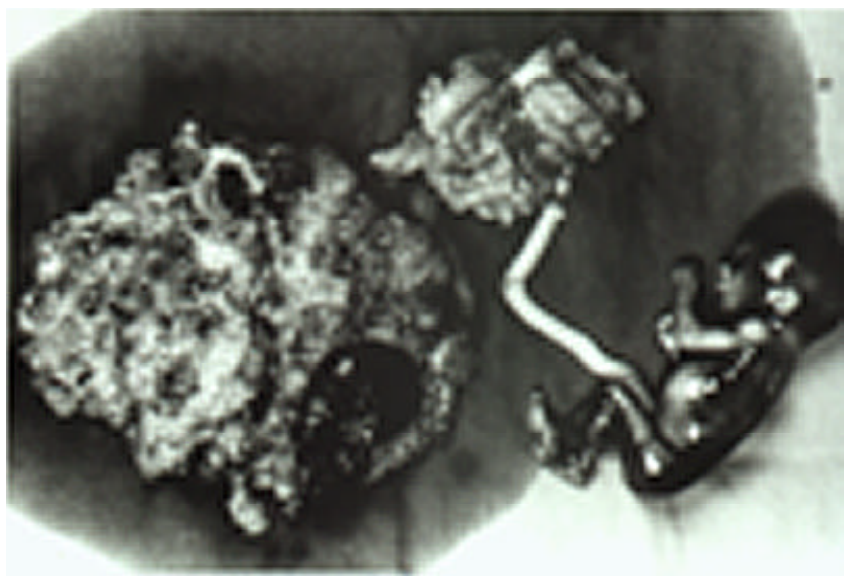

Figura 3- Gestação gemelar: placenta molar ao lado de feto e placenta normais.

No $4^{\circ}$ dia de pós-operatório, a paciente apresentou pressão arterial de 140/90 mmHg e nível de $\beta$ hCG de $130.290 \mathrm{mUI} / \mathrm{ml}$. O ultra-som de controle evidenciou útero vazio (volume de 719 $\mathrm{cm}^{3}$ ), miométrio homogêneo, cistos teca-luteínicos bilateralmente com diâmetro superior a $10 \mathrm{~cm} \mathrm{e}$ baixa resistência em ambas as artérias uterinas. O escore prognóstico dessa gravidez molar revelou alto risco para o desenvolvimento de TTG, em decorrência do grande volume uterino, níveis elevados de $\beta$ hCG e cistos tecaluteínicos volumosos, sendo que a paciente foi acompanhada no ambulatório semanalmente. A pressão arterial técnicas na desparafinização não permitiram número suficiente de células e a presença de debris celular impediu a determinação do índice de DNA. Entretanto, a análise do conteúdo de DNA das células visualizadas foi sugestiva de tumor diplóide.

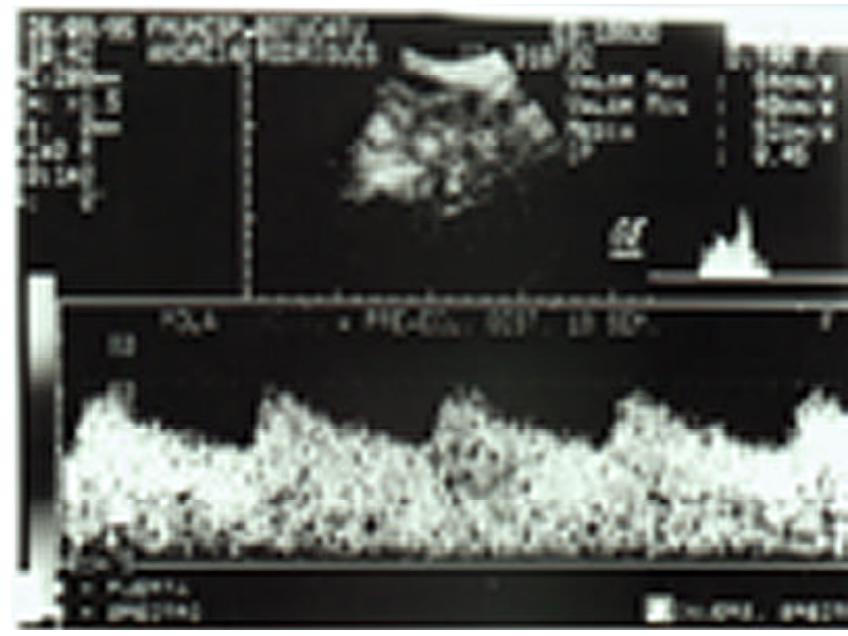

Figura 2- Dopplerfluxometria da artéria uterina revelando baixa resistência ao fluxo.

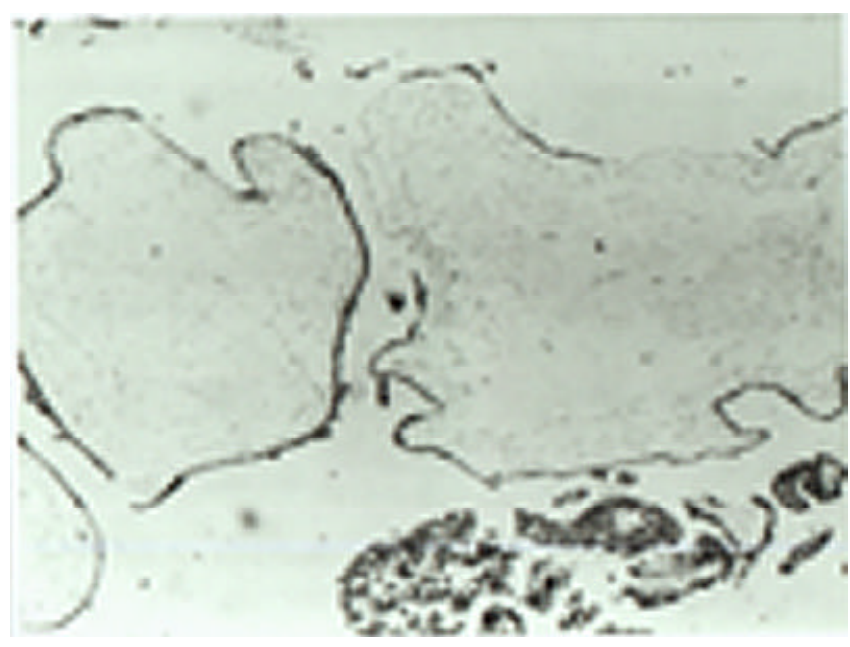

Figura 4- Histopatológico de mola hidatiforme completa com grande vilosidade hidrópica avascular e áreas com hiperplasia trofoblástica exuberante (HE 100X).

normalizou no $8^{\circ}$ dia após o esvaziamento molar. Entretanto, observou-se persistência dos cistos tecaluteínicos, apesar da paciente estar assintomática. A curva de regressão do $\beta$ hCG mostrou um plateau da $2^{\mathrm{a}}$ a $4^{\mathrm{a}}$ semana com níveis de $90.000 \mathrm{mUI} / \mathrm{ml}$ após o esvaziamento molar e o ultra-som revelou útero de $675 \mathrm{~cm}^{3}$, miométrio heterogêneo e hipervascularizado, proliferação tecidual heterogênea intra-uterina, cistos tecaluteínicos bilateralmente (ovário direito - 171 $\mathrm{cm}^{3}$; ovário esquerdo $-187 \mathrm{~cm}^{3}$ ) e circulação uterina com resistência diminuída $(\mathrm{PI}=0,41)$. A análise da evolução clínica, laboratorial e ultra-sonográfica 
levou ao diagnóstico de TTG. Investigação de mestástases com raio-X de tórax e ultra-som abdominal resultou negativa. Optou-se pelo uso da monoquimioterapia com metotrexato (MTX), administrado diariamente na dose de $0,4 \mathrm{mg} / \mathrm{kg}$ de peso, por via intramuscular, durante 5 dias consecutivos. Foram realizadas quatro séries desse esquema em intervalos mínimos de 7 e máximos de 14 dias, entre elas. A resposta à quimioterapia foi adequada. Houve diminuição significativa dos níveis de $\beta$ hCG. Concomitantemente, houve involução uterina e regressão dos cistos tecaluteínicos, embora mantivesse dopplerfluxometria com baixa resistência em ambas as artérias uterinas (Tabela 1).
Anteriormente a cada ciclo de quimioterapia, foram avaliados o peso da paciente, função de medula óssea, renal e hepática e $\beta$ hCG. Após a $4^{\mathrm{a}}$ série de monoquimioterapia (MTX) manifestou-se toxicidade: eritema polimorfo e mucosite grave, sendo necessário o uso de ácido folínico, antibióticos e fungicidas. Houve rápida recuperação do estado geral e optou-se pela mudança do esquema quimioterápico para metotrexato com resgate com ácido folínico, em regime ambulatorial. Nos dias 1, 3, 5 e 7 utilizou-se MTX na dose de 1,0 $\mathrm{mg} / \mathrm{kg}$ de peso por via intramuscular e nos dias 2 , 4, 6 e 8 ácido folínico na dose de $0,1 \mathrm{mg} / \mathrm{kg}$ também por via intramuscular. A paciente realizou 6 ciclos desse esquema e não apresentou qualquer efeito normal das dosagens séricas de bhCG.

Tabela 1 - Parâmetros de resposta ao tratamento com MTX.

\begin{tabular}{|c|c|c|c|c|c|c|c|c|c|c|}
\hline Parâmetros $M T X-s$ & $1^{\mathrm{a}}$. & $2^{\mathrm{a}}$ & $3^{\mathrm{a}}$ & $4^{\mathrm{a}}$. & $5^{\mathrm{a}}$ & $6^{a}$ & $7^{\mathrm{a}}$ & $8^{a}$ & $9^{a}$ & $10^{\mathrm{a}}$. \\
\hline$\beta h C G \mathrm{mUI} / \mathrm{ml}$ & 90.000 & 13.184 & 5.875 & 1.734 & 734 & 86 & 53 & 18 & 17 & 20 \\
\hline Volume uterino $\left(\mathrm{cm}^{3}\right)$ & 675 & 402 & 309 & 220 & 220 & - & 224 & - & - & 120 \\
\hline Ovário D $\left(\mathrm{cm}^{3}\right)$ & 171 & 27 & 8 & 5 & 5 & - & 7 & - & - & 5 \\
\hline Ovário $\mathrm{E}\left(\mathrm{cm}^{3}\right)$ & 187 & 187 & 48 & 9 & 10 & - & 9 & - & - & 9 \\
\hline Art. Uterina D (PI)* & 0,41 & - & - & 0,78 & - & - & 0,60 & - & - & 0,55 \\
\hline Art. Uterina E (PI)* & 0,54 & - & - & 0,83 & - & - & 0,53 & - & - & 0,60 \\
\hline
\end{tabular}

*PI = Índice de pulsatilidade

\section{Discussão}

Existe dificuldade no diagnóstico de $\mathrm{MC}$ em gestação gemelar e a chave desse diagnóstico é o cariótipo do tecido molar. Esse exame diferencia a MC de origem androgenética da MP triplóide, de origem genética materna e paterna. A ultrasonografia obstétrica pode interpretar erroneamente como MP uma gravidez gemelar com $\mathrm{MC}$ e feto coexistentes, porque em ambos os casos, há existência de feto e placenta normal ao lado de alteração hidrópica do tecido viloso. Entretanto, o ultra-som auxiliou o diagnóstico em vários casos citados na literatura 14,16. No caso relatado, foi realizado o diagnóstico de gestação múltipla com MC e feto coexistentes no momento da resolução da gestação: características macroscópicas da MC de "cachos de uva" e a presença de feto sem malformações, cordão umbilical e placenta normais. Em decorrência de hemorragia uterina maciça, realizaram-se a técnica da vácuoaspiração, seguida pela extração manual do feto e da placenta, por via transvaginal. O parto com histerotomia piora o prognóstico materno aumentando o risco de doença trofoblástica persistente $^{1,13}$. O estudo histopatológico do tecido molar retirado foi compativel com os critérios histológicos de Szulman e Surti ${ }^{15}$ para o diagnóstico de MC. A citometria de fluxo, apesar do número insuficiente de células, revelou conteúdo de DNA diplóide na placenta molar.

Pacientes com gravidez gemelar, na qual coexistem feto normal e MC, são de alto risco para desenvolver TTG. Foram relatadas porcentagens variando de 45 a 63\% de malignização nesses $\operatorname{casos}^{2,3,14}$. Em contraste, apenas de 14 a $20 \%$ das pacientes com mola completa em gestação única desenvolvem TTG ${ }^{9,10,14}$. Essa paciente evoluiu para TTG e necessitou de múltiplas séries de quimioterapia para obter remissão completa da doença.

O manejo da gestação gemelar com MC e feto coexistente depende das circunstâncias de cada caso e uma conduta conservadora pode ser possível $^{12}$. A conduta deve ser individualizada, sendo que a vitalidade fetal, o desejo da paciente em continuar a gravidez e suas condições clínicas é que devem nortear a decisão do obstetra.

Relatamos nossa experiência clínica para 
promover melhor entendimento da história natural da gestação gemelar com MC e feto coexistentes. Consideramos esses casos, um desafio clínico, em decorrência de sua propensão para desenvolver complicações clínicas e TTG.

\section{SUMMARY}

Twin pregnancy in which a normal fetus and a complete mole develop at the same time is a rare event. Clinical complications and malignancy are frequent in this type of disease. This report is about a case of a late diagnosis due to the presence of the fetus. The diagnosis was made when the pregnancy was interrupted and then confirmed by histopathological study and flow cytometry. The pregnancy was terminated transpelvically due to massive uterine hemorrhage. The postmolar follow-up showed the persistence of high levels of bhCG. The patient's complete recovery was achieved after the administration of methotrexate. The diagnosis, natural history, and procedures for this rare disease are discussed in view of this case.

KEY WORDS: Gestational trophoblastic disease. Twin pregnancy. Complete hydatidiform mole. Pregnancy complications. Hemorrhage. Chemotherapy. Ultrasonography.

\section{Referências}

1.Belfort P, Madi JM. Neoplasia trofoblástica gestacional. In: Halbe $H$. editor Tratado de ginecologia. São Paulo: Roca; 1987. cap. 52, p. 640-9.

2.Bristow RE, Shumway JB, Khouzami AN, Witter FR. Complete hydatidiform mole and surviving coexistent twin. Obstet Gynecol Surv, 1996; 51: 7059.

3.Choi-Hong SR, Genest DR, Crum CP, Berkowitz R, Goldstein DP, Schofield DE. Twin pregnancies with complete hydatidiform mole and coexisting fetus: use of fluorescent in situ hybridization to evaluate placental $\mathrm{X}$-and-Y - chromosomal con- tent. Hum Pathol 1995; 26: 1175-80.

4.Cunningham FG, Mc Donald PC, Gant NF, Leveno KJ, Gilstrap LC, Hankins GDV, Clark SL. Diseases and abnormalities of the placenta. In: Williams Obstetrics $20^{\text {th }}$ edition. Norwalk: Prentice - Hall, 1997, cap. 30, p. 669-91.

5.Driscoll SG. Problems and pitfalls in the histopathologic diagnosis of gestational trophoblastic lesions. J Reprod Med 1987; 32: 632-8.

6.Goldstein DP, Berkowitz RS, Bernstein MR. Management of molar pregnancy. J Reprod Med, 1981; 26: 208-12.

7.Jacobs PA, Wilson CM, Sprenkle JA, Rosenshein NB, Migeon BR. Mechanism of origin of complete hydatidiform moles. Nature 1980; 286: 714-6.

8.Kajii T, Ohama K. Androgenetic origin of hydatidiform mole. Nature 1977; 268: 633-4.

9.Lurain JR, Brewer JI, Torok, EE, Halpern B. Natural history of hydatidiform mole after primary evacuation. Am J Obstet Gynecol 1983; 145: 591-5.

10.Maestá I, Rudge MVC, Michelin OC, Calderon IMP, Costa CA, Parreira DJR, et al. Aspectos clínicos, evolutivos e terapêuticos da doença trofoblástica gestacional. J Bras Ginecol 1995; 105: 243-8.

11. Miller D, Jackson R, Ehlen T, McMurtrie E. Complete hydatidiform mole coexistent with a twin live fetus: clinical course of four cases with complete cytogenetic analysis. Gynecol Oncol 1993; 50: 119-23.

12.Paradinas FJ, Fisher RA, Browne P, Newlands ES. Diploid hydatidiform moles with fetal red blood cells in molar villi 1 - Pathology, incidence and prognosis. J Pathol 1997; 181: 183-8.

13.Schlaerth JB. Methodology of molar pregnancy termination. Clin Obstet Gynecol 1984; 27 : 192-8.

14.Steller MA, Genest DR, Bernstein MR, Lage JM, Goldstein DP, Berkowitz RS. Natural history of twin pregnancy with complete hydatidiform mole and coexisting fetus. Obstet Gynecol 1994; 83: 35-42.

15.Szulman AE, Surti U. The syndrome of hydatidiform mole. I. Cytogenetic and morphologic correlations. Am J Obstet Gynecol 1978; 131: 665-71.

16.Vejerslev LO. Clinical management and diagnostic possibilities in hydatidiform mole with coexistent fetus. Obstet Gynecol Surv 1991; 46: 577-88. 